\title{
Photogrammetrische Verfahren in der bildgebenden Photometrie
}

\author{
Christian Schrader, Armin Sperling \\ Physikalisch-Technische Bundesanstalt \\ Arbeitsgruppe 4.12 Photometrie \\ Bundesallee 100, D-38116 Braunschweig
}

\section{Zusammenfassung}

Diese Arbeit beschreibt die Anwendung photogrammetrischer Methoden zur Bestimmung der geometrischen Aufnahmekonfiguration bei der Durchführung photometrischer Messungen mit Leuchtdichtemesskameras. Das Ziel ist es, die orts- und richtungsabhängigen Eigenschaften der beteiligten Komponenten detaillierter zu beschreiben, als gegenwärtig üblich. Hierzu ist es notwendig, Messungen mit verschiedenen Untersuchungsobjekten oder Kameras verlässlich zueinander in Beziehung zu setzen, sei es zur Trennung von verschiedenen sich überlagernden Eigenschaften oder aber zur Beobachtung der zeitlichen Stabilität. In diesem Paper werden nach der Vorstellung des Messaufbaus die photogrammetrischen Grundprinzipien kurz erläutert und die Anpassungen und Besonderheiten für den vorliegenden Aufbau diskutiert. Weiterhin werden die ersten Messungen zur Separierung der orts- und richtungsabhängigen Eigenschaften vorgestellt.

Keywords: Photometrie, Photogrammetrie, Leuchtdichte, Messkamera

\section{Problemstellung}

In den letzten Jahren gewannen Messkameras zur Messung der Leuchtdichte von Objekten zunehmend Bedeutung zur schnellen ortsaufgelösten Bestimmung von Leuchtdichteverteilungen. Für die PTB als nationales Metrologieinstitut besteht zukünftig die Aufgabe, diese Kameras rückführbar unter Angabe einer beigeordneten Messunsicherheit zu kalibrieren, was bisher noch nicht möglich ist.

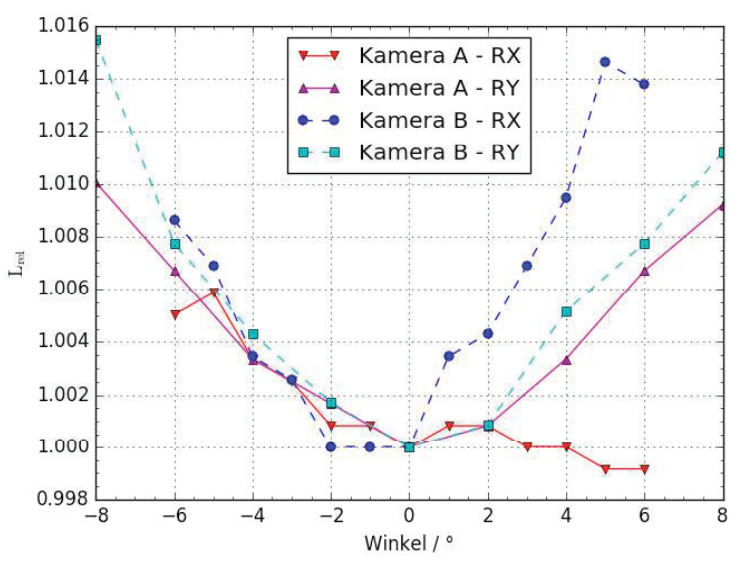

Abb 1: Relative Richtungsabhängigkeit der gemessenen Leuchtdichte aufgrund der Lambert-Idealisierung während der Kalibrierung
Im Vergleich zu herkömmlichen Leuchtdichtemessgeräten mit nur einem Photometer steigt bei Verwendung eines Bildsensors als Photometermatrix die Komplexität des Messprozesses deutlich an. Für die Ermittlung der Umrechnungsfaktoren vom zur Beleuchtungsstärke proportionalen Sensorsignal jedes Pixels zu Leuchtdichte wird das Bild eines ausgedehnten Leuchtdichtenormals (LDN) aufgenommen. Dieses wird hierbei idealisiert als homogener Lambert-Strahler angesehen, d.h. orts- und richtungsabhängige Variationen werden nicht berücksichtigt, was zu Abweichungen bei der Bestimmung der Kalibrierfaktoren und somit zu Messunsicherheiten führt. Abb. 1 zeigt exemplarisch die Auswirkungen für zwei Leuchtdichtemesskameras, bei denen die Abstrahlrichtung der beobachteten Region eines LDN konstant gehalten wird, während die Kamera zwischen den Messungen horizontal und vertikal gedreht wird. Im Bild wandert die Auswerteregion dabei zu den Bildrändern. Es kommt hier zu einer Überhöhung der gemessenen Leuchtdichte aufgrund des richtungsabhängigen Leuchtdichteabfalls während der Kalibrierung. Anstatt einem zu erwartenden näherungsweise quadratischen Verlauf zeigt sich auch eine deutliche Asymmetrie.

Verallgemeinert lässt sich sagen, dass sich in den aufgenommenen Bildern die orts- und richtungsabhängigen, spektralen und zeitlichen 
Inhomogenitäten von Kamera (Sensor, $V_{\lambda^{-}}$ Filter, Objektiv) und Leuchtdichtenormal überlagern.

\section{Aufgabe und Lösungsansatz}

Das Ziel ist es, diese orts- und richtungsabhängigen Inhomogenitäten von LDN und Kamera getrennt zu bestimmen, um diese Daten zur Bestimmung der Messunsicherheit verwenden zu können. Hierzu soll die Kamera frei vor einem LDN bewegt werden und somit Beobachtungsort und -richtung der Messungen im relevanten Bereich gezielt variiert werden können. Für die Auswertung ist es erforderlich, für jeden Bildpunkt den zugehörigen beobachteten Objektpunkt eindeutig zuordnen zu können. Hierdurch wird es möglich, die Daten aus verschiedenen Messungen zueinander in Beziehung zu setzen. Dieses wird notwendig um die Inhomogenitäten zu separieren, aber auch, um die zeitliche Stabilität der Komponenten (Alterung) beobachten zu können.

Die für die Zuordnung von Objekt- und Bildpunkt relevanten Größen sind zu bestimmen. Für die Wahl des Verfahrens war es wichtig, dass dieses auch mit verschiedensten Kameras, Objektiven und Messobjekten nach jeder Konfigurationsänderung durchgeführt werden kann. Eine Ausrichtung anhand äußerer Merkmale, z.B. Gehäusekanten, ist somit nicht praktikabel. Die Abmessungen der zu untersuchenden Leuchtdichtenormale liegen im Bereich von $20 \mathrm{~mm}$ bis $500 \mathrm{~mm}$.

Die Wahl fiel daher auf die Nahbereichsphotogrammetrie, die einen hochflexiblen und hochgenauen Methodensatz zur Bestimmung der gesuchten Parameter bereitstellt. Sie erlaubt eine weitgehende Automatisierung der geometrischen Einmessung und verspricht somit eine geringe subjektive Beeinflussung durch den Durchführenden.

\section{Aufbau des Messplatzes}

Der Messplatz besteht in der aktuellen Konfiguration aus einem fünfachsigen Positioniersystem mit drei translatorischen und zwei rotatorischen Achsen. Der Arbeitsraum beträgt $\pm 250 \mathrm{~mm}$ in horizontaler und $\pm 160 \mathrm{~mm}$ in vertikaler Richtung. Die Rotationachsen schneiden sich in einem Punkt und erlauben eine Drehung der Kamera um $\pm 90^{\circ}$. Vor dem Positioniersystem befindet sich das LDN. Abb. 2 zeigt den Aufbau. Das photogrammetrische Referenzmuster vor der Ausgangsöffnung des LDN dient der geometrischen Einmessung und ist reproduzierbar montierbar. Das aktuell verwendete LDN hat eine Austrittsöffnung mit $70 \mathrm{~mm}$ Durchmesser.

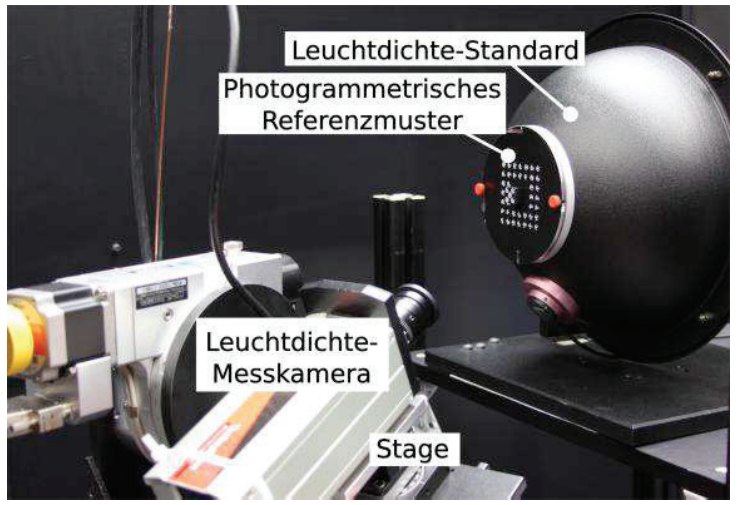

Abb 2: Aufbau des Messystems, zu sehen sind das Leuchtdichtenormal mit photogrammetrischem Referenzmuster, Stage mit Kamera und einer Rotationsachse

Für Drehungen um die Ausgangsöffnung des LDN sind translatorische Bewegungen der Kamera notwendig, die proportional zur Fokusentfernung sind. Der hierbei nutzbare Winkelbereich ist somit begrenzt durch den zur Verfügung stehenden translatorischen Arbeitsraum der Achsen und dem Kollisionsbereich zwischen Stage und Montageplattform des LDN. Weiterhin muss auch die Schärfentiefe der Kamera berücksichtigt werden.

Der hier verwendete Aufbau dient als Versuchsplattform für die Entwicklung des generellen Verfahrens und besteht aus einer Kombination aus einzelnen motorisierten Verfahrachsen, die in serieller Kinematik kombiniert wurden. Zur Verbesserung der Positioniergenauigkeit und Steigerung der Flexibilität ist zukünftig eine Umrüstung auf ein mechanisch besser ausgelegtes Positioniersystem mit drei Rotationsachsen vorgesehen. Die zusätzliche Achse bringt eine höhere Flexibilität und niedrigeren Ausrichtungsaufwand, bedeutet jedoch eine zusätzliche Positionierunsicherheit. Entsprechend dem Betrachtungsabstand wirken sich Unsicherheiten der Rotationsachsen stark auf die anvisierten Objektpunkte aus. Für eine ausgewogene Auslegung müssen jedoch die Anforderungen durch das Messverfahren selbst genauer spezifiziert sein, so dass Rahmenbedingungen wie z.B. Fokusentfernung, Sichtfeldgröße, Kameragewicht und Arbeitsraum entsprechend berücksichtigt werden können. Evtl. wird es sinnvoll sein, die photometrischen Messungen mit photogrammetrischen Korrekturmessungen der Orientierung zu kombinieren. 


\section{Konzept zur Einmessung der geometri- schen Konfiguration}

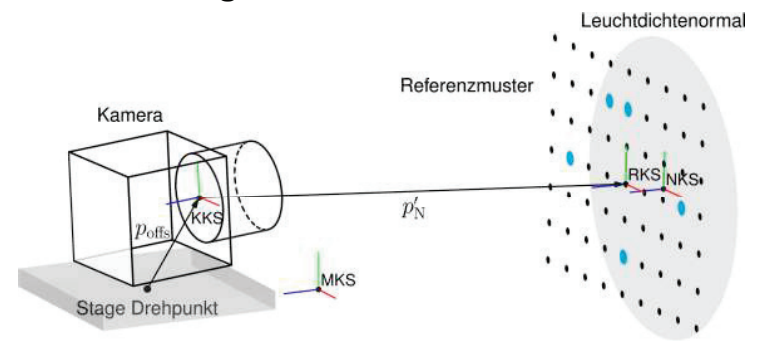

\section{Abb 3: $\quad$ Gesuchte Größen der Einmessung}

In Abb. 3 ist der prinzipielle Aufbau des Systems mit zugordneten Koordinatensystemen und den gesuchten geometrischen Parametern gezeigt. Die für die Positionierung der Kamera relevante Größe ist die Position ihres Projektionszentrums. Über dieses lässt sich die Abbildung der Kamera besonders einfach beschreiben. Die Lage des Projektionszentrums zu äußeren Referenzflächen der Kamera ist jedoch im Allgemeinen nicht bekannt und zudem noch von der Fokuseinstellung des Objektivs abhängig. Für die Kamera wird ein Kamerakoordinatensystem (KKS) definiert, dessen Ursprung im Projektionszentrum (PZ) liegt. Seine $X / Y$-Achsen seien parallel zum Bildsensor und die Z-Achse zeigt in Richtung der optischen Achse zum Bildsensor.

In Abb. 3 ist angedeutet, dass das KKS relativ zum natürlichen Drehpunkt der Rotationsachsen verschoben ist und aufgrund von Ausrichtungsfehlern zu den Achsen des globalen Maschinenkoordinatensystems (MKS) verdreht ist. Diese relative Pose (äußere Orientierung) kann durch jeweils sechs Parameter beschrieben werden (drei für Translation, drei für die Euler-Winkel der Rotation), welche zu bestimmen sind.

Für die reproduzierbare Lokalisierung der gemessenen Daten muss in der Austrittsöffnung durch die Festlegung eines Koordinatensystems eine Referenzebene definiert werden. $\mathrm{Da}$ die Austrittsöffnung naturgemäß keine markanten Eigenschaften hat, die sich zur Festlegung des Koordinatensystems eignen, wird vor der Austrittsöffnung ein optisch kooperatives Referenzmuster angebracht, dessen Lage gut bestimmt werden kann. Bei ausreichend guter Reproduzierbarkeit der Anbringung besteht zwischen der Lage des Referenzmusters (RKS) und dem Koordinatensystem des Normals (NKS) ein konstanter Offset, so dass es genügt, die relative Pose dieses Referenzmusters zur Kamera zu bestimmen und den Offset dann zu addieren.

Da einem Punkt auf der Referenzfläche des LDN eindeutig ein Bildpunkt zugeordnet wer- den soll, müssen ebenfalls die Parameter eines geeigneten Abbildungsmodels, die sogenannte innere Orientierung der Kamera, bestimmt werden (mindestens drei Parameter: Kamerakonstante, Hauptpunktlage, Verzeichnungsparameter).

\section{Prinzip der Photogrammetrie}

Zur Bestimmung der geometrischen Konfiguration werden Verfahren der Nahbereichsphotogrammetrie verwendet. Im Folgenden sollen diese kurz zusammengefasst werden. Für Detailinformationen und mathematische Herleitungen sei auf die umfangreiche Literatur in $[1,2,3]$ verwiesen.

Unter Photogrammetrie versteht man eine Gruppe von Methoden zur Ableitung von Informationen zur Lage oder Form von Objekten aus ein oder mehreren Bildern. Hierbei werden in der beobachteten Szene Strukturen (Targets) benötigt, die sich in verschiedenen Bildern eindeutig identifizieren lassen. Deren Bildkoordinaten werden möglichst genau bestimmt. Den Targets werden Positionen im Objektraum zugeordnet, die im Allgemeinen nicht bekannt sind. Über ein Abbildungsmodell der Kamera werden diese Positionen mit der inneren und äußeren Orientierung verknüpft und auf theoretische Bildkoordinaten abgebildet. Auf diese Weise lässt sich ein nichtlineares Gleichungssystem (GLS) aufstellen, dessen Lösung die unbekannten Parameter für eine möglichst hohe Übereinstimmung von theoretischen und beobachteten Bildkoordinaten liefert.

Diese Methode zeichnet sich durch hohe Flexibilität aus. Zum einen lassen sich weitere Eigenschaften des Systems durch zusätzliche Gleichungen berücksichtigen, was hier für die Ermittlung der Offsetpose zur Anwendung kommt. Desweiteren lassen sich Vorkenntnisse über einzelne Parameter, die z. B. aus anderen Messungen stammen, über eine $\mathrm{Ge}$ wichtung während der Lösung des Gleichungssystems berücksichtigen. D. h. ohne strukturelle Änderungen ist das Verfahren an verschiedene Messaufgaben anpassbar. Die einzelnen Teilschritte sollen im Folgenden unter Einbeziehung der lokalen Besonderheiten näher erläutert werden.

\section{Photogrammetrischer Workflow}

Der erste Schritt besteht in der Festlegung von identifizierbaren Merkmalen, deren Position sich gut bestimmen lässt. Identifikation und Positionsbestimmung müssen in weitem $\mathrm{Be}$ reich unabhängig von Abbildungsmaßstab, Rotation, Unschärfe, perspektivischer Verzerrung und Beleuchtungsgegebenheiten sein. 
Besonders bewährt haben sich hierfür schwarz-weiße Kreismarken. Die Identifizierung kann z.B. über die Relativlage zu charakteristischen Targets (Abb. 3: große Punkte in planarem Raster) erfolgen oder durch zusätzlich angebrachte Codierungen. Im vorliegenden Aufbau werden Ring-Codes verwendet, wodurch die Targets freier platziert werden können und auch dreidimensionale TargetAnordnungen möglich sind (Abb. 4). Diese sind aufgrund der Tiefeninformation nummerisch vorteilhaft. Die genaue Anordnung der Targets ist im Prinzip nicht relevant, was die Anpassung an unterschiedliche Aufnahmeentfernungen und Messobjekte erleichtert. Es sollte jedoch das Bildfeld, über alle Aufnahmepositionen betrachtet, weitgehend überdeckt werden. Außerdem ist der Abstand zweier Punkte zu bestimmen und als bekannter Maßstab in das Gleichungssystem als zusätzliche Randbedingung aufzunehmen.

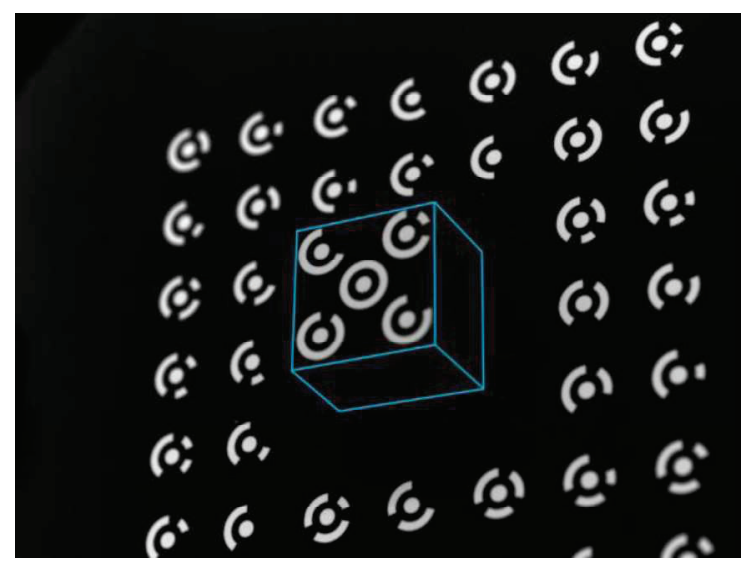

Abb 4: Referenzmuster zur geometrischen Einmessung, Markenabstand $8 \mathrm{~mm}$, zentraler Quader mit erhöhten Marken hervorgehoben

Der für die Einmessung verwendete Bildverband, besteht zum einen aus sechs zur zentralen Aufnahmeposition leicht versetzten Bildern und zum anderen aus neun Bildern mit um das LDN rotierter Aufnahmeposition (Abb. 5). Die Drehpunktlage kann für die Einmessung grob geschätzt werden. Es ist nicht erforderlich, dass das Referenzmuster auf jedem Bild vollständig erfasst wird.

Als nächstes ist ein Kameramodell zu wählen. Die Basis bildet hierbei die Zentralprojektion, die um zusätzliche Terme erweitert werden kann um verschiedene Aberrationen, wie z.B. radialsymmetrische Verzeichnungen oder richtungsabhängigen Abbildungsmaßstab zu modellieren. Bisher ist im vorliegenden Aufbau nur die einfache Zentralprojektion implementiert.

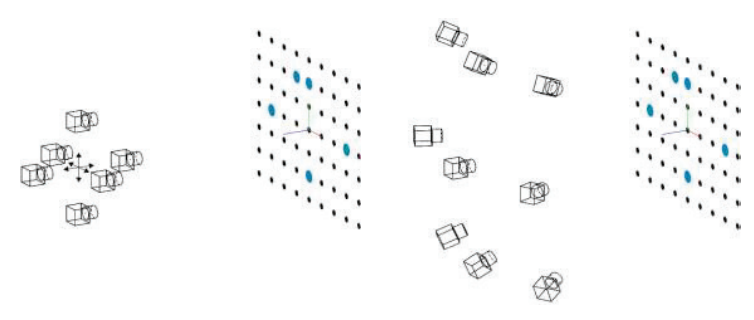

Abb 5: Aufnahmepositionen der geometrischen Einmessung

Die zu minimierende Residuumsfunktion des Gleichungssystems besteht aus den Differenzen zwischen beobachteter und theoretischer Bildkoordinate jedes Punktes in jedem Bild (zwei Gleichungen pro Beobachtung für x- und $y$-Koordinate) und zusätzlichen Gleichungen für die Randbedingungen wie dem oben erwähnten bekannten Punktabstand und Gleichungen für die Offsetpose der Kamera. Letztere berechnen sich nach folgendem Ansatz. Es sei $\mathbf{C}$ die Matrix einer Pose in homogenen Koordinaten:

$\mathbf{C}=\left[\begin{array}{cc}\mathbf{R} & \mathbf{T} \\ 0 & 1\end{array}\right]$

bestehend aus dem Translationsvektor $\mathbf{T}$ und der Rotationmatrix $\mathbf{R}$ als Funktion der drei Eulerwinkel (entsprechend der kinematischen Verknüpfungsreihenfolge des Aufbaus). Dann ist die Kamerapose gegeben durch die zur Aufnahmeposition gehörige Stagepose $\mathbf{C}_{\text {stage }}$ und die konstante Offsetpose $\mathbf{C}_{\text {offset }}$ und es muss für alle Aufnahmepositionen gelten:

$$
\mathbf{C}_{\text {cam }} \stackrel{!}{=} \mathbf{C}_{\text {stage }} \cdot \mathbf{C}_{\text {offset }} \text {. }
$$

Aus der Rotationskomponente des Produkts der rechten Seite lassen sich die zugehörigen Eulerwinkel berechnen. Zusammen mit der translatorischen Komponente ergeben sich die Parameter einer theoretischen äußeren Kameraorientierung, deren Differenzen zu den bereits im Gleichungssystem enthaltenen Parametern der äußeren Orientierung als zusätzliche Gleichungen dem Gleichungssystem hinzugefügt werden. Hierdurch ergibt sich auch gleich die Anbindung des gesamten Verbandes aus Punkt- und Kamerapositionen an das übergeordnete Maschinenkoordinatensystem, so dass dieses nicht zusätzlich festgelegt werden muss bzw. darf.

Die Lösung des nichtlinearen Gleichungssystems erfolgt durch Ausgleichung nach vermittelnden Beobachtungen (Bündel-Block-Ausgleich). Für den iterativen Ausgleichsalgorithmus werden geeignete Näherungswerte für die unbekannten Parameter benötigt. Für die äu- 
ßeren Orientierungen der Kamera werden die Stagepositionen der Aufnahmen verwendet, die X/Y-Targetpositionen sind aus der Druckvorlage bekannt und werden um die grob geschätzte Z-Position erweitert.

Problematisch ist die gleichzeitige Bestimmung der inneren Orientierung während des Ausgleichs (Simultankalibrierung). Es bestehen starke Korrelationen zwischen der inneren und äußeren Orientierung der Kamera, die zu instabilem Lösungsverhalten führen. Dieses lässt sich durch spezielle Aufnahmekonfigurationen mit stark gekanteten (um die optische Achse gedrehten) Kameraorientierungen verhindern, jedoch kann das aktuell verwendete Positioniersystem diese Bewegung nicht ausführen. Deshalb wird die innere Orientierung der Kamera nach [4] und [3], S. $115 \mathrm{ff}$., aus der perspektivischen Verzeichnung orthogonaler Vektorenpaare berechnet. Zur Auswertung werden nur die stark geneigten Aufnahmen der Eckpositionen (Abb. 5 rechts) verwendet, da nur diese die geforderte perspektivische Verzeichnung aufweisen. Das Referenzmuster besteht deshalb entgegen der oben erwähnten freien Targetanordnung aus einem regelmäßigen Gitter, um diese orthogonalen Vektorenpaare zu liefern. Zur Erhöhung der Genauigkeit werden zudem nicht nur drei Vektorenpaare ausgewertet, sondern mehrere aus mehreren Bildern, so dass im Gegensatz zu den obigen Quellen ein überbestimmtes lineares Gleichungssystem aufgebaut werden kann, dessen Least-Square-Lösung dann als innere Orientierung verwendet wird und mit hohem Gewicht, jedoch nicht fix, im Ausgleich berücksichtigt wird.

Mit der Lösung des Gleichungssystems sind alle geometrischen Parameter bekannt, so dass im Folgenden die Kamera relativ zu einem gegebenen Punkt positioniert werden kann. Für Neigungswinkel von bis zu $20^{\circ}$ und einem Abstand der Kamera vom LDN von $200 \mathrm{~mm}$ liegen die lateralen Positionsabweichungen des betrachteten Punktes unter $\pm 0,4 \mathrm{~mm}$. was der 2,5-fachen Schrittweite der Rotationsachsen entspricht und sich somit nahe der mechanischen Auflösungsgrenze bewegt.

\section{Bestimmung der Inhomogenitäten}

Im Folgenden werden die Ansätze zur Bestimmung der Inhomogenitäten erläutert und erste Messergebnisse vorgestellt. Zur Trennung der richtungsabhängigen Eigenschaften von LDN und Kamera wird diese so bewegt, dass sich für einen betrachteten Sichtstrahl möglichst nur eine Eigenschaft signifikant ändert. Hierbei werden nur Kamerabilder ohne

Umrechnung in Leuchtdichte aufgenommen. Es wird jeweils ein fester Punkt auf dem LDN beobachtet. Die Drehungen erfolgen dabei um zwei senkrechte Achsen, die parallel zur Referenzebene des LDN sind, üblicherweise die Xund Y-Achse. In Abb. 6 sind die Bewegungsmuster für eine Achse skizziert.

a)

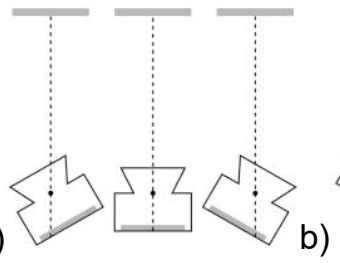

Abb 6: $\quad$ Variation der relativen Ausrichtung

a) Drehung um Projektionszentrum

b) Drehung um Punkt auf Normal

Die Bildkoordinate erhält man aus dem räumlichen Rückschnitt des Objektpunktes mittels Kameramodell, der ermittelten inneren Orientierung und der zur Aufnahmeposition gehörenden äußeren Orientierung. Zur Reduktion des Rauschens wird an Stelle eines einzelnen Pixelwertes eine Region symmetrisch um den Bildpunkt festgelegt (hier $41 \times 41$ Pixel), über die gemittelt wird.

\section{Richtungsabhängigkeit der Kamera}

Für eine Drehung um das Projektionszentrum der Kamera (Abb. 6a) variiert nur die Durchstrahlungsrichtung der Kamera, die Abstrahlrichtung des LDN bleibt konstant. Als Ergebnis erhält man die Richtungsabhängigkeit der Kamera, bestehend aus dem theoretisch rotationssymmetrischen Anteil der Vignettierung zuzüglich lokaler Störungen.

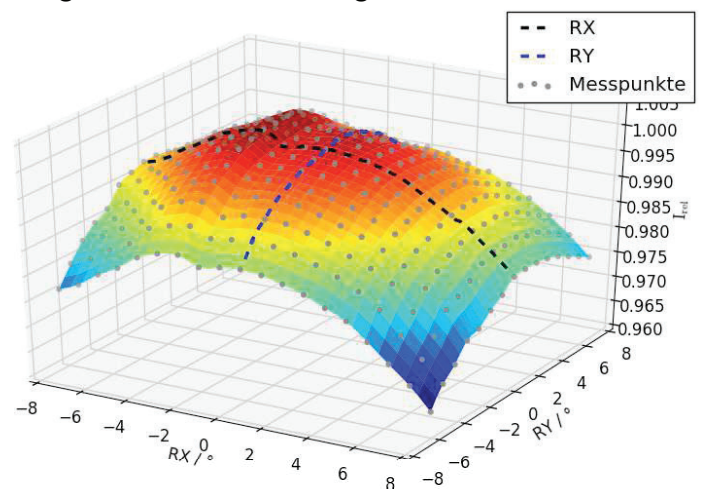

Abb 7: Relative Richtungsabhängigkeit der Kamera

Abb. 7 zeigt die Richtungsabhängigkeit der Kamera als Ergebnis der Messung bei Drehung um das Projektionszentrum. Die Neigungswinkel betragen $\pm 8^{\circ}$ bzw. $\pm 6^{\circ}$ und sind so gewählt, dass entsprechend der Bildgröße 
die Auswerteregionen immer vollständig im Bild liegen. Der beobachtete Punkt lag in der Mitte des LDN. Durch die gemessenen Punkte ist zur Veranschaulichung eine interpolierte Fläche gelegt. Die Werte sind normiert auf den Wert bei senkrechter Beobachtungsrichtung $\left(0^{\circ}, 0^{\circ}\right)$.

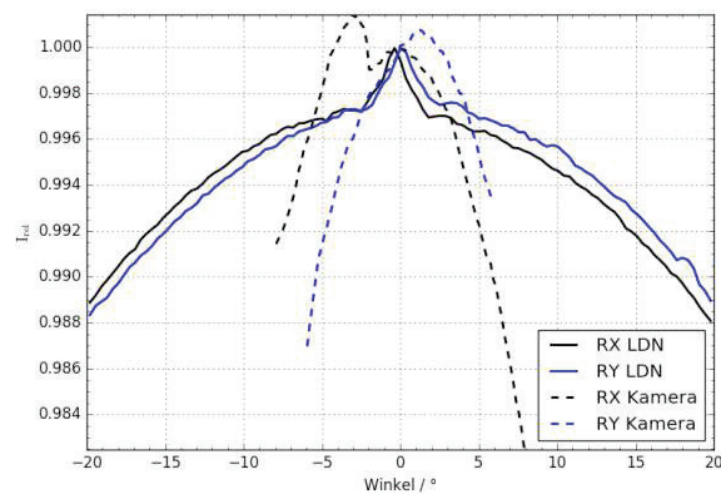

Abb 8: Relative Richtungsabhängigkeit von Kamera und Leuchtdichtenormal, nur einachsige Drehung

Zusätzlich zu den flächenhaften Messpunkten mit niedriger Auflösung wurden zwei höher aufgelöste jeweils einachsige Scans aufgenommen (schwarz/blau), um die Feinstruktur exemplarisch besser auflösen zu können. Diese Scans sind in gleicher Farbe in Abb. 8 dargestellt. Gegenüber einem erwarteten Maximalwert von 1 bei $0^{\circ}$ zeigen sich unerwartete Überhöhungen seitlich der Nullage. Diese drehen sich auch bei um $90^{\circ}$ gedrehter Kamera entsprechend mit, so dass diese gesichert als Kameraeigenschaften einzuordnen sind. Vermutete Ursache hierfür sind Interreflektionen im Objektiv oder am $V_{\lambda}$-Filter.

\section{Richtungsabhängigkeit des Leuchtdichte- normals}

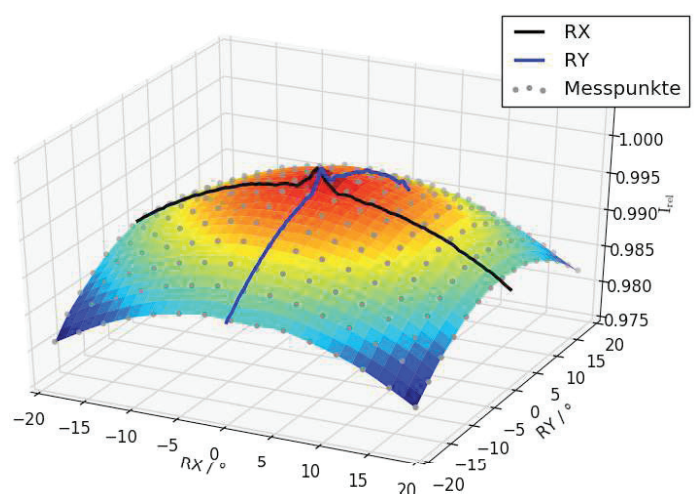

Abb 9: Relative Richtungsabhängigkeit des Leuchtdichtenormals im Zentrum der Austrittsfläche
Liegt der Drehpunkt gemeinsam mit dem beobachteten Objektpunkt auf der Referenzebene des LDN, dann wird dieser Objektpunkt für alle Aufnahmepositionen auf denselben Bildpunkt abgebildet. Hierbei ändert sich nur die Abstrahlrichtung des LDN, die Durchstrahlrichtung der Kamera bleibt konstant. Man erhält somit die reine Richtungsabhängigkeit des LDN an dem beobachteten Ort.

Abb. 9 zeigt die Richtungsabhängigkeit des LDN für einen gewählten Winkelbereich von $\pm 20^{\circ}$ und identischem Beobachtungspunkt wie bei der vorherigen Messung. Wie zuvor sind auch hier einachsige Scans aufgetragen, die ebenfalls in Abb. 8 dargestellt sind. Es ist ein sehr gleichmäßiger Abfall zu größeren Winkeln hin zu verzeichnen, jedoch zeigt sich im Bereich von $\pm 2^{\circ}$ eine lokale Signalspitze von ca. $0,3 \%$. Dieser Effekt zeigte sich auch bei einer vergleichbaren Messung mit einem konventionellen Leuchtdichtemessgerät. Als Ursache werden Reflektionen zwischen dem Objektiv und der Streuscheibe des LDN angesehen. Alle in Abb. 8 gezeigten Verläufe sind auf denselben Referenzwert normiert, der separat bei $0^{\circ}$ ermittelt wurde. Die Abweichung der Verläufe vom Idealwert 1 bei $0^{\circ}$ (identische Aufnahmeposition) ist somit ein Kriterium für die zeitliche Stabilität der Messungen.

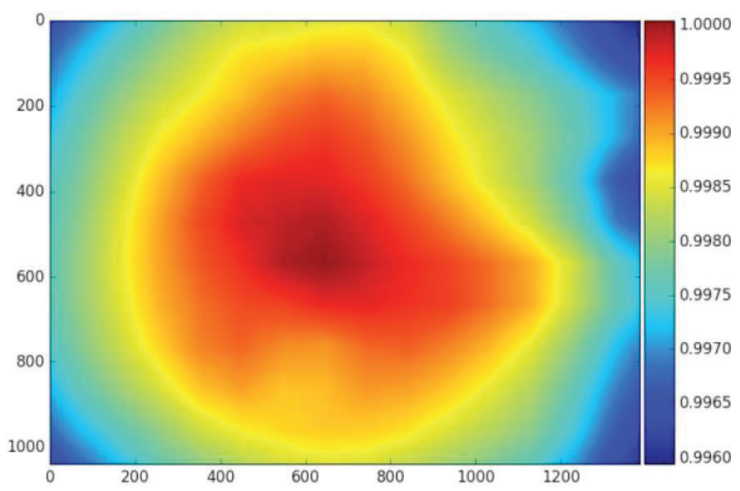

Abb 10: Korrekturfunktion der relativen Richtungsabhängigkeit des Leuchtdichtenormals

Unter der Annahme einer über die Fläche konstanten Richtungsabhängigkeit lassen sich aus den Daten in Abb. 9 Korrekturfunktionen berechnen (Abb. 10), indem für jeden Pixel die kamerainterne Richtung über die äußere Kameraorientierung in die LDN-bezogene Richtung umgerechnet wird und die zugehörige Abschwächung interpoliert wird.

Soll die Richtungsabhängigkeit über der Referenzebene des LDN ortsaufgelöst erfasst werden, kann man diese Messung mit variablen Betrachtungsort wiederholen, was jedoch aufgrund der hohen Messzeit 
problematisch ist. In Abb. 11 wurde dieses exemplarisch für 30 Punkte durchgeführt. Die einzelnen Flächen sind auf ihr jeweiliges Maximum bei $0^{\circ}$ skaliert. Bis auf einen Messpunkt sind alle Verläufe sehr homogen und liegen in einem Band von 0,15\%.

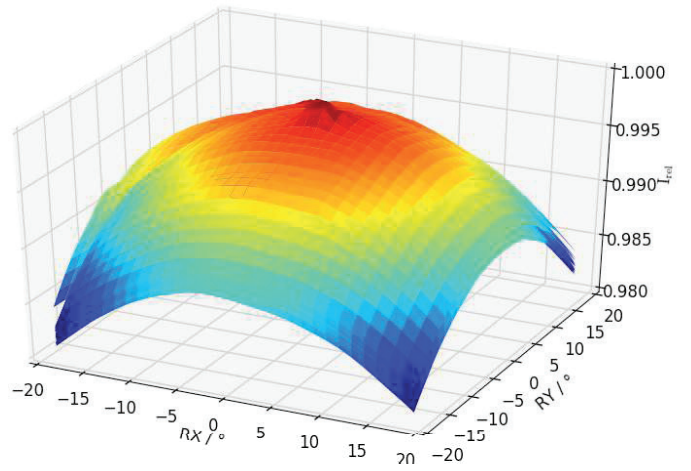

Abb 11: Relative Richtungsabhängigkeit des LDN an 29 Punkten der Austrittsfläche

Zur Beschleunigung der Messung ist hier vorgesehen, an Stelle eines Sichtstrahles die gesamten Bilddaten auszuwerten, so dass mit dem Scan für einen Punkt entsprechend Abb. 9 die Daten für große Teile der LDN-Austrittsöffnung bestimmt werden können. Jedes Bild wird hierbei gleichmäßig in Auswerteregionen unterteilt wobei der Mitte dieser Region ein Sichtstrahl mit Richtungswinkeln zugeordnet werden kann. Für diese Richtung ergibt sich aus der Richtungsbhängigkeit der Kamera (Abb. 7) ein Korrekturfaktor für den gemittelten Wert der Auswerteregion. Aus dem photogrammetrischen Vorwärtsschnitt auf die Referenzebene kann dem Messwert eine Position zugeordnet werden. Man erhält somit für viele Positionen auf der Referenzebene einen Messwert und eine zugeordnete Richtung. Aufgrund der perspektivischen Verzerrung sind diese Punkte ungleichförmig auf der Referenzebene verteilt, so dass diese in Regionen unterteilt werden muss, innerhalb derer die Richtungen und Messwerte zusammengefasst werden und aus diesen Daten für die mittlere Position eine Interpolationsfläche der Richtungsempfindlichkeit entsprechend Abb. 9 berechnet werden kann. Die Auswertung hat Parallelen zu der von Nahfeldgoniophotometern.

Alternativ kann auch umgekehrt diskretisiert werden. Hierbei werden auf der Referenzebene des LDN Punkte in regelmäßigem Raster festgelegt, diese auf das Bild zurückgeschnitten und um diese zentralen Punkte eine Region gemittelt. Beide Arten der photogrammetrischen Parallelisierung werden ge- genwärtig umgesetzt. Nach der Abb. 10 gezeigten Messung ist jedoch absehbar, dass das derzeit verwendete LDN u. U. zu homogen ist, um die parallelisierte Auswertung validieren zu können. Hier sind geeignete Änderungen vorzunehmen, um eine signifikante Variationen der Richtungsabhängigkeit zu erhalten.

\section{Hauptkomponentenanalyse}

In [5] wurde ein Verfahren vorgestellt, mit dem die örtlichen Inhomogenitäten von Kamera und Normal separiert werden können. Hierzu wird ein Satz von Bildern mit leichter inhaltlicher Variation durch Hauptkomponentenanalyse (engl. PCA) in Eigenbilder zerlegt. Durch Linearkombination dieser Eigenbilder lässt sich das Originalbild verlustfrei rekonstruieren. Dem Eigenbild mit dem größten Eigenwert werden hierbei die Kameraeigenschaften zugeordnet. Im Gegensatz der niedrigen Auflösung der oben gezeigten Winkelscans liefert die PCA pixelaufgelöste Daten, berücksichtigt jedoch nicht die Richtungsabhängigkeit des LDN.

In [5] wurde hierbei der Schwerpunkt auf die Bestimmung der Inhomogenität des LDN gelegt und das Kameraeigenbild nicht für die Rekonstruktion verwendet. Hier soll hingegen auch die Kamerainhomogenität mitbestimmt, um sie mit den zuvor ermittelten Daten kombinieren zu können. Die Kameraeigenschaften sollen dabei nicht verändert werden. Deshalb werden nur laterale Verschiebungen mit konstanter Aufnahmeentfernung durchgeführt.
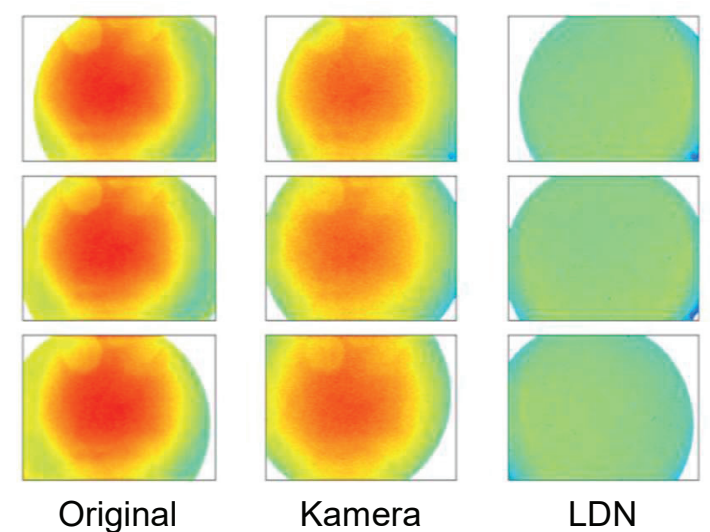

Abb 12: Zerlegung von Bildern mittels PCA in Kamera- und LDN-Komponente mit unvollständiger Bildüberdeckung

Abb. 12 zeigt für drei von neun Bildern eines hier verwendeten Bildsatzes das Originalbild, und die separierten Anteile von Kamera und LDN. Man erkennt, dass das Bildfeld nicht vollständig vom LDN ausgefüllt wird. Diese äußeren Bereiche wurden maskiert und für die PCA mit dem Bildmittelwert aufgefüllt. Hier 
lässt sich die Kenntnis der Abbildungseigenschaften zum Stitching der einzelnen Datensätze an ihren Rändern verwenden, so dass man eine vollständige Beschreibung der Kamera bzw. des LDN erhält.

\section{Zusammenfassung und Ausblick}

Es wurde ein auf der Nahbereichsphotogrammetrie beruhendes Verfahren vorgestellt, mit dem die geometrische Konfiguration eines kamerabasierten Photometriemessplatzes flexibel und mit hoher Genauigkeit bestimmt werden kann. Es ermöglicht es durch eine wiederholbare dreidimensionale Lokalisierung, bisher notwendige Vereinfachungen in der Systemmodellierung zu überwinden und bildet die Basis für eine orts- und richtungsaufgelöste Bestimmung der photometrischen Eigenschaften der beteiligten Komponenten. Es wurden Ansätze für diese Messungen gezeigt, die darauf beruhen, mit den Daten der photogrammetrischen Einmessung eine eindeutige Zuordnung von Objekt- und Bildpunkt durchführen zu können.

Für die photogrammetrische Einmessung ist die Robustheit der Bestimmung der inneren Kameraorientierung zu verbessern. Gegebenenfalls ist diese mit einem speziellen Referenzmuster in einem separaten Schritt durchzuführen. Im Bereich der photometrischen Auswertung besteht die nächste Aufgabe in der Verwendung der vollständigen Bildinformation anstelle einer einzelnen Messregion. Weiterhin muss untersucht werden, ob sich die Interreflektionen reduzieren lassen, bzw. wie sie sich auf die weitere Auswertung auswirken, insbesondere bei der Verknüpfung von Messdaten aus Messreihen mit unterschiedlicher Stärke der Interreflektionen. Hier werden geeignete Referenzwerte (vgl. Wert im Nullpunkt von Abb. 8) benötigt, die sich zuverlässig bestimmen lassen. Außerdem ist eine weitere Wissensbasis über die zu erwartenden und somit zu berücksichtigenden Eigenschaften aufzubauen, indem systematische Messreihen mit unterschiedlichen Komponenten (Kameras, Objektive, Normale) durchgeführt werden.

\section{Literaturnachweis}

[1] Luhmann T., Nahbereichsphotogrammetrie, 2010, Wichmann Verlag, Berlin

[2] Kraus, Photogrammetrie - Band 1, 7. Aufl., 2004, Walter de Gruyter Verlag, Berlin, ISBN 3-11-017708-0

[3] Kraus K., Photogrammetrie - Band 2, 3. Aufl. 1996, Dümmlers Verlag, Bonn, ISBN 3-427-78653-6
[4] Novak K., Orientierung von Amateuraufnahmen ohne Paßpunkte, S. 44-50,

Geowissenschaftliche Mitteilungen 28, 1986, Institut für Photogrammetrie, Wien

[5] Ferrero, A.; Lopez, M.; Campos, J. \& Sperling, A. Spatial characterization of cameras for lowuncertainty radiometric measurements Metrologia, 2014, 51, 316 